\title{
Generalized MMSE beamforming for multicell MIMO systems with random user geometry and channel feedback latency
}

\author{
Illsoo Sohn ${ }^{1}$, Hyoungjoo Lee ${ }^{2}$ and Kwang Bok Lee $3^{3 *}$
}

\begin{abstract}
In this paper, we propose a generalized minimum mean square error (MMSE) beamforming for downlink multicell multiple-input multiple-output (MIMO) systems with local BS cooperations. Unlike the previous beamforming strategies which have been designed for an idealized multicell MIMO system model, we consider a complicated but realistic multicell MIMO system model. Our realistic multicell MIMO system model captures (i) different average SNRs of users due to random geometrical distribution of users within cells, (ii) channel feedback latency due to air propagation time and signal processing time, and (iii) different channel aging effects for intracell channel information and intercell channel information due to an additional backhaul latency during the exchange of information between pairs of neighboring cells. The key novelty of this paper is that we derive the closed-form beamforming expressions of the generalized MMSE beamforming using convex optimization technique. The closed-form beamforming expression gives insights on how the geometry of users and channel feedback latency affect the construction of optimal beamforming vectors. Numerical results verify that the proposed generalized MMSE beamforming outperforms previous beamforming strategies in both BER and average throughput performances.
\end{abstract}

Keywords: Multiple-input multiple-output (MIMO); Multicell MIMO; Minimum mean square error (MMSE) beamforming; Limited backhaul

\section{Introduction}

With increasing demands of various multimedia service, the next generation wireless communication systems are expected to support much higher data rate than before. The demand prompts an increase of interests in spectrally efficient technologies and therefore multiple-input multiple-output (MIMO) has been extensively studied as a key technology for cellular systems. Recently, MIMO techniques are severely challenged by intercell interference. In [1-3], intercell interference has been shown to significantly degrade system performance especially in the reusability of the spectral resource, which results in limiting the overall spectral efficiency.

To mitigate the degradation, a promising approach is to allow the base stations (BSs) to cooperate. The BS

\footnotetext{
*Correspondence: klee@snu.ac.kr

3 Department of Electrical and Computer Engineering and INMC, Seoul National University, 599 Gwanak-ro, Gwanak-Gu, Seoul 110-799, Korea Full list of author information is available at the end of the article
}

cooperation was introduced in [4] and then extended to downlink cooperative MIMO systems [5,6]. In downlink cooperative MIMO systems, multiple BSs exploit their multiple antennas and use beamforming techniques based on the sharing of all required information including user data and channel state information (CSI). The BS cooperation has been theoretically proven to improve the system performance in terms of cell coverage, celledge user throughput as well as average sum rate, and the improvement becomes much larger as the network size grows. However, the BS cooperation suffers from real-world constraints such as limited capacity and nonnegligible latency of the backhaul network for information sharing and huge computational complexity of joint processing across all BSs.

Several pragmatic solutions for the problems have been suggested in [7-9]. The key idea behind these techniques is sharing CSI locally between neighboring cells without sharing user data. In multicell MIMO systems with local CSI sharing, each BS determines its beamforming vector 
based on CSI of both intracell and intercell links. In [7], a simple beamforming technique for maximizing user's signal-to-noise ratio (SNR) has been introduced. And zero-forcing beamforming (ZFBF) has been extended to multicell MIMO systems in [8]. A beamforming technique that computes the best beamforming vector by defining a new metric called signal-to-generating interference-plusnoise ratio (SGINR) has also been proposed for multicell MIMO systems in [9].

The aforementioned beamforming techniques, however, consider a simplified multicell MIMO system model assuming an ideal user distribution within a cell and an unlimited-capacity and zero-latency backhaul network. In the emerging cellular systems where an early form of coordinated multipoint transmission and reception (CoMP) is ready for commercial deployments [10], the following implementation issues should be carefully considered. Firstly, cellular users are randomly located within their cells and therefore experience quite different path loss $[11,12]$. As a result, all users now have different values of average SNR. Secondly, when a mobile station (MS) receives a reference signal from a $\mathrm{BS}$, it requires processing time to perform channel estimation and create CSIs for channel feedback. Similarly, a BS also requires processing time to collect CSI feedback and compute beamforming vectors after MSs feedbacks their CSIs. A typical time delay between the channel estimation and actual transmission via beamforming can increase up to $8 \mathrm{~ms}$ in commercialized long term evolution (LTE) systems. This channel aging effect due to the channel feedback latency significantly degrades beamforming performances as discussed in many related literatures. Finally, the backhaul network, which is specifically defined as X2-link in commercialized LTE systems, imposes some limitations for the exchanges of CSIs between pairs of BSs. The backhaul network introduces additional channel feedback latencies across cells, which further increases channel aging effects [13]. Thus, the shared CSI collected from neighboring cells are normally more outdated than that of the self cell and thus provides relatively inaccurate information for the determination of beamforming vectors.

In this paper, we consider the design of downlink beamforming technique for multicell MIMO systems with local CSI sharing. In particular, we follow the principle of MMSE beamforming that computes the best beamforming vectors by minimizing the error between the transmitted signal and received signal caused from interference and noise as in [14-16] and generalize it for multicell MIMO systems considering real-world implementation issues. The beauty of the proposed MMSE beamforming is that we can find the closed form beamforming expressions using convex optimization techniques even for the complicated realistic multicell MIMO system model [17]. Numerical results verify that the proposed generalized MMSE beamforming outperforms other beamforming strategies in terms of both BER and average throughput.

The notations used in this paper are as follows: Boldfaces are used for vectors and matrices; $\mathbb{E}\{\cdot\}$ denotes a mathematical expectation of random variables; $\mathbf{I}_{M}$ denotes a $M$-by- $M$ identity matrix; superscripts * and * are used to denote complex conjugate and optimal value, respectively.

The rest of this paper is organized as follows: Section 2 describes our realistic multicell MIMO system model. In Section 3, we briefly provide an overview of three conventional beamforming strategies developed for downlink multicell MIMO systems with local CSI sharing. In Section 4, we formulate an optimization problem and derive a closed-form solution of the proposed generalized MMSE beamforming. Finally, we provide numerical results in Section 5 and conclude in Section 6.

\section{System model}

Figure 1 illustrates a downlink multicell MIMO system model. Total $L$ cells are deployed in the network. The BS in each cell is located in the center with $M$ transmit antennas and each user with a single receive antenna is randomly distributed within the corresponding cell. During the downlink transmissions, users experience intercell interference from neighboring cells. For simplicity, we assume that $M$ users in each cell are already selected by a user scheduler at the given time. Therefore, each BS serves $M$ selected users in the corresponding cell simultaneously.

The received signal of the $k$ th user in the $m$ th cell is represented as

$$
\begin{aligned}
y_{k, m}= & \sqrt{\rho_{k, m}}\left(\mathbf{h}_{k, m}^{*} \mathbf{w}_{k, m}\right) x_{k, m} \\
& +\sqrt{\rho_{k, m}} \sum_{i=1, i \neq k}^{M}\left(\mathbf{h}_{k, m}^{*} \mathbf{w}_{i, m}\right) x_{i, m} \\
& +\sum_{j=1, j \neq m}^{L} \sum_{i=1}^{M} \sqrt{\eta_{k, m, j}}\left(\mathbf{g}_{k, m, j}^{*} \mathbf{w}_{i, j}\right) x_{i, j}+n_{k, m},
\end{aligned}
$$

where $x_{k, m}$ is the data symbol of the $k$ th user transmitted through $M$ transmit antennas of the $m$ th BS satisfying $\mathbb{E}\left[\left|x_{k, m}\right|^{2}\right]=1 . \mathbf{h}_{k, m}$ and $\mathbf{g}_{k, m, j}$ denote the desired timevarying channel vector from the $m$ th BS towards the $k$ th user in the $m$ th cell $\left(\mathbf{h}_{k, m} \in \mathbb{C}^{M \times 1}\right)$ and the interfering time-varying channel vector between the $k$ th user in the $m$ th cell and the $j$ th BS, respectively. $x_{k, m}$ and the elements of both $\mathbf{h}_{k, m}$ and $\mathbf{g}_{k, m, j}$ are assumed to be independent and identically distributed (i.i.d.) circularly symmetric complex Gaussian random variables with zero 


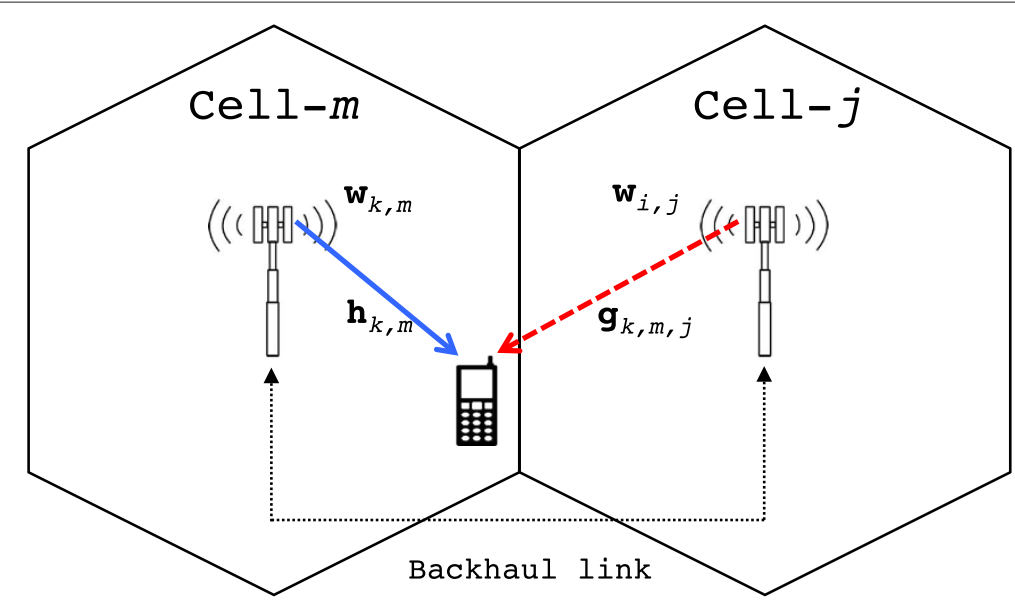

Figure 1 Multicell MIMO system model.

mean and unit variance. $\mathbf{w}_{k, m}$ is the unit-norm beamforming vector for the transmission to the $k$ th user in the $m$ th cell $\left(\mathbf{w}_{k, m} \in \mathbb{C}^{M \times 1}\right)$, and $n_{k, m}$ is the additive complex Gaussian noise with zero means and unit variance. $\rho_{k, m}$ is the average signal-to-noise ratio (SNR) reflecting random geometry as $\rho_{k, m}=\frac{P}{M N_{0} d_{k, m}{ }^{\alpha}}$, where $d_{k, m}, \alpha, N_{0}$, and $P$ denote the distance of the $k$ th user in the $m$ th cell from the $m$ th BS, path loss exponent, noise power, and transmit power, respectively. Similarly, $\eta_{k, m, j}$ is the average interference-to-noise ratio (INR) for the interference that the $j$ th BS causes to the $k$ th user in the $m$ th cell, denoted as $\eta_{k, m, j}=\frac{P}{M N_{0} d_{k, m, j}{ }^{\alpha}}$ where $d_{k, m, j}$ is the distance of the $k$ th user in the $m$ th cell from the $j$ th BS. In (1), the first term on the right implies a desired signal, the second term implies an intracell interference from the same cell, and the third term implies an intercell interference leaked from the neighboring cells.

In downlink multicell MIMO systems with local CSI sharing, MSs need to acquire channel information such as $\mathbf{g}_{k, m, j}$ and $\eta_{k, m, j}$ by monitoring reference signals from neighboring cells. After the MSs report the information to their own BSs with channel feedback, neighboring BSs share it through the backhaul network. It has been proven in $[18,19]$ that the first-order Gauss-Markov process model well describes relatively small delays in the communication systems. Using a first-order GaussMarkov process, channel aging effects can be modeled as

$$
\begin{gathered}
\mathbf{h}_{k, m}=\varepsilon_{k, m} \cdot \mathbf{h}_{k, m}^{\left(\tau_{k, m}\right)}+\sqrt{1-\varepsilon_{k, m}^{2}} \cdot \mathbf{h}_{\mathrm{w}}, \\
\mathbf{g}_{k, m, j}=\varepsilon_{k, m, j} \cdot \mathbf{g}_{k, m, j}^{\left(\tau_{k, m, j}\right)}+\sqrt{1-\varepsilon_{k, m, j}^{2}} \cdot \mathbf{g}_{\mathrm{w}},
\end{gathered}
$$

where $\mathbf{h}_{k, m}^{\left(\tau_{k, m}\right)}$ and $\mathbf{g}_{k, m, j}^{\left(\tau_{k, m, j}\right)}$ are respectively CSIs of $\tau_{k, m}$ and $\tau_{k, m, j}$ time ago when CSIs had been created at the MS for channel feedback. Including both air propagation time and signal processing time, $\tau_{k, m}$ implies the intracell feedback latency from the $k$ th user in the $m$ th cell to the $m$ th BS and $\tau_{k, m, j}$ implies the intercell feedback latency from the $k$ th user in the $m$ th cell to the $j$ th BS. Practically, $\tau_{k, m}<\tau_{k, m, j}$ since $\tau_{k, m, j}$ additionally captures exchange time of channel information between the BSs over the backhaul link in addition to intracell feedback latency [13]. $\mathbf{h}_{\mathrm{w}}$ and $\mathbf{g}_{\mathrm{w}}$ denotes the uncorrelated components with $\mathbf{h}_{k, m}$ and $\mathbf{g}_{k, m, j}$, and consist of i.i.d. circular symmetric complex Gaussian random variables with zero mean and unit variance. The scalar values of $\varepsilon_{k, m}$ and $\varepsilon_{k, m, j}$ are the temporal correlation coefficients for the desired and interfering channel, respectively, and can be characterized by

$$
\begin{aligned}
\varepsilon_{k, m} & =J_{0}\left(2 \pi f_{D} \tau_{k, m}\right), \\
\varepsilon_{k, m, j} & =J_{0}\left(2 \pi f_{D} \tau_{k, m, j}\right),
\end{aligned}
$$

where $J_{0}(x)$ is the zero ${ }^{\text {th }}$-order Bessel function and $f_{D}$ is the maximum Doppler frequency in Hertz. As shown in [19], the maximum Doppler frequency can be represented by $f_{D}=\frac{v_{k, m} f_{c}}{c}$ where $v_{k, m}$ is the velocity of the $k$ th user in the $m$ th cell, $f_{c}$ is the carrier frequency, and $c$ is the speed of light, respectively.

\section{Beamforming strategies for downlink multicell MIMO systems}

In this section, three beamforming strategies are presented, which have been originally developed for the multiuser MIMO in a single-cell system model. Motivated by the analogies between mitigating intracell and intercell interference except the heterogeneity of interference links, three beamforming strategies are generalised to fit in our multicell system model with slight modifications. In addition, limitations of the conventional approaches are discussed in detail. 


\subsection{Maximum SNR (MAX-SNR) Beamforming Strategy}

In downlink multicell MIMO systems, the simplest beamforming strategy is to choose the transmit beamforming vector which maximizes the signal power of the user without regards to the generating interference to other users [7]. Therefore, in the maximum SNR beamforming strategy, the beamforming vector of the $k$ th user in the $m$ th cell is simply chosen as

$$
\mathbf{w}_{k, m}^{\star}=\underset{\mathbf{w}_{k, m} \in \mathbb{C}^{M \times 1}}{\arg \max }\left\|\mathbf{h}_{k, m}^{*} \mathbf{w}_{k, m}\right\|^{2}
$$

Note that the maximum SNR beamforming strategy has an advantage in enabling the decentralized network control without information sharing on the backhaul link. However, the system performance could be significantly degraded by intercell interference especially for near celledge users.

\subsection{Minimum generating interference (MIN-GI) beamforming strategy}

As an opposite approach to MAX-SNR beamforming strategy, each BS can find the transmit beamforming vectors to minimize the generating interference to other users [8] by solving the following objective problem:

$$
\begin{aligned}
\mathbf{w}_{k, m}^{\star}= & \underset{\mathbf{w}_{k, m} \in \mathbb{C}^{M \times 1}}{\arg \min }\left(\sum_{i=1, i \neq k}^{M}\left\|\mathbf{h}_{i, m}^{*} \mathbf{w}_{k, m}\right\|^{2}\right. \\
& \left.+\sum_{i=1}^{M} \sum_{j=1, j \neq m}^{L}\left\|\mathbf{g}_{i, j, m}^{*} \mathbf{w}_{k, m}\right\|^{2}\right) .
\end{aligned}
$$

Note that the transmit beamforming vectors chosen in (7), called minimum generating interference (MIN-GI) beamforming, are recognized as an extension of zeroforcing beamforming (ZFBF) since they try to avoid interference both among users and between cells. Inheriting the drawbacks of ZFBF [20,21], the MIN-GI beamforming strategy suffers from severe performance degradation especially in the time-varying channel, where reported CSI becomes quickly outdated.

\subsection{Maximum signal-to-generating interference-plus-noise ratio (MAX-SGINR) beamforming strategy}

As a compromise of the MAX-SNR beamforming strategy and the MIN-GI beamforming strategy, an efficient beamforming method for downlink multicell MIMO systems with local CSI sharing is introduced in [9], called the maximum signal-to-generating interferenceplus-noise ratio (MAX-SGINR) beamforming. In the
MAX-SGINR beamforming strategy, the beamforming vector of the $k$ th user in the $m$ th cell is determined as:

$$
\mathbf{w}_{k, m}^{\star}=\underset{\mathbf{w}_{k, m} \in \mathbb{C}^{M \times 1}}{\arg \max } \frac{\left\|\mathbf{h}_{k, m}^{*} \mathbf{w}_{k, m}\right\|^{2}}{1+\sum_{i=1, i \neq k}^{M}\left\|\mathbf{h}_{i, m}^{*} \mathbf{w}_{k, m}\right\|^{2}+\sum_{i=1}^{M} \sum_{j=1, j \neq m}^{L}\left\|\mathbf{g}_{i, j, m}^{*} \mathbf{w}_{k, m}\right\|^{2}} .
$$

Note that the numerator of (8) is the signal power at the desired user and the denominator consists of noise and interference to other users generated by the $m$ th BS. In [9], it has been shown that in the simplified multicell-MIMO environment where all users have the same SNR and INR, MAX-SGINR beamforming outperforms both MAX-SNR beamforming and MIN-GI beamforming.

\subsection{Limitations of conventional multicell MIMO beamforming strategies}

In [7-9], the conventional multicell MIMO beamforming strategies does not consider realistic cellular parameters such as the random geometry of the users and different temporal correlations due to the mobility of the users. In [12], it is proven that user's different SNR and INR reflecting the distances from the BSs significantly affect system performance. Therefore, the beamforming vector for multicell MIMO systems should be effectively chosen according to the different user SNR-INR ratio. In addition, it has been shown that the accuracy of channel information dominates the beamforming performance of multicell MIMO systems [14], and thus channel feedback latency as well as user mobility play critical roles in determining beamforming vectors. The MMSE beamforming forms a favorable mathematical framework to capture aforementioned considerations as will be shown in the next section.

\section{Generalized MMSE beamforming for downlink multicell MIMO systems}

\subsection{Problem formulation}

The mean square error (MSE) of the $k$ th user in the $m$ th cell which is conditioned by realistic multicell MIMO system model parameters can be defined as

$$
\begin{aligned}
\operatorname{MSE}_{k, m}= & \mathbb{E}_{x_{k, m}, \mathbf{h}_{\mathrm{w}}, \mathbf{g}_{\mathrm{w}}}\left[\frac{1}{\rho_{k, m}}\left|y_{k, m}-\sqrt{\rho_{k, m}} \cdot x_{k, m}\right|^{2} \mid\right. \\
& \left.\times \varepsilon_{k, m}, \varepsilon_{k, m, j}, \rho_{k, m}, \eta_{k, m, j}, \mathbf{h}_{k, m}^{\left(\tau_{k, m}\right)}, \mathbf{g}_{k, m, j}^{\left(\tau_{k, m}\right)}\right],
\end{aligned}
$$

where $\mathbb{E}_{x_{k, m}, \mathbf{h}_{\mathrm{w}}, \mathbf{g}_{\mathrm{w}}}$ is the conditional expectation of $x_{k, m}$, $\mathbf{h}_{\mathrm{w}}$, and $\mathbf{g}_{\mathrm{w}}$. Note that in (9), we modify the conventional MSE definition, $\mathrm{MSE}_{k, m}=\mathbb{E}\left[\left|y_{k, m}-x_{k, m}\right|^{2}\right]$, due to the difference between our system model and the conventional multicell MIMO system model [7-9], where, for simplicity, all average SNRs are assumed to be the same without considering the random geometry of users. 
However, our complicated system model considers the different received average SNR $\rho_{k, m}$, and INR $\eta_{k, m, j}$ that capture the effects of both transmit power and path loss. Thus, the modified MSE definition reflecting the difference of those system models is used for the design of beamforming vectors.

Theorem 1. With the realistic multicell MIMO system model in (1) capturing random geometry of MSs and channel feedback latency including backhaul network, the mean square error of the received signal at the kth MS in the mth cell is given as

$$
\begin{aligned}
\operatorname{MSE}_{k, m}= & \varepsilon_{k, m}^{2} \mathbf{w}_{k, m}^{*} \mathbf{h}_{k, m}^{\left(\tau_{k, m}\right)} \mathbf{h}_{k, m}^{\left(\tau_{k, m}\right) *} \mathbf{w}_{k, m}+\left(1-\varepsilon_{k, m}^{2}\right) \\
& \cdot \mathbf{w}_{k, m}^{*} \mathbf{w}_{k, m}+\varepsilon_{k, m}^{2} \\
& \cdot \sum_{j=1, j \neq k}^{M} \mathbf{w}_{j, m}^{*} \mathbf{h}_{j, m}^{\left(\tau_{j, m}\right)} \mathbf{h}_{j, m}^{\left(\tau_{j, m}\right) *} \mathbf{w}_{j, m}+\left(1-\varepsilon_{k, m}^{2}\right) \\
& \cdot \sum_{j=1, j \neq k}^{M} \mathbf{w}_{j, m}^{*} \mathbf{w}_{j, m}+\frac{1}{\rho_{k, m}} \cdot \sum_{j=1, j \neq m}^{L} \sum_{i=1}^{M} \varepsilon_{k, m, j}^{2} \\
& \cdot \eta_{k, m, j} \cdot \mathbf{w}_{i, j}^{*} \mathbf{j}_{i, j, m}^{\left(\tau_{i, j}\right)} \mathbf{g}_{i, j, m}^{\left(\tau_{i, j}\right) *} \mathbf{w}_{i, j}+\frac{1}{\rho_{k, m}} \\
& \cdot \sum_{j=1, j \neq m}^{L} \sum_{i=1}^{M}\left(1-\varepsilon_{k, m, j}^{2}\right) \cdot \eta_{k, m, j} \\
& \cdot \mathbf{w}_{i, j}^{*} \mathbf{w}_{i, j}-\varepsilon_{k, m} \cdot \mathbf{w}_{k, m}^{*} \mathbf{h}_{k, m}^{\left(\tau_{k, m}\right)}-\varepsilon_{k, m} \\
& \cdot \mathbf{h}_{k, m}^{\left(\tau_{k, m}\right) *} \mathbf{w}_{k, m}+\frac{\mathbf{w}_{k, m}^{*} \mathbf{w}_{k, m}}{\rho_{k, m}}+1 .
\end{aligned}
$$

Proof. See details in the Appendix.

From Equation 10, the beamforming vectors need to be determined to minimize the total MSEs under the constraint of BS power. Therefore, the MMSE-based optimization for multicell MIMO systems can be formulated as the following problem:

$$
\begin{aligned}
\underset{\mathbf{w}_{1,1}, \cdots, \mathbf{w}_{M, L} \in \mathbb{C}^{M \times 1}}{\operatorname{minimize}} & \sum_{m=1}^{L} \sum_{k=1}^{M} \operatorname{MSE}_{k, m}, \\
\text { subject to } & \left\|\mathbf{w}_{1,1}\right\|^{2}=\cdots=\left\|\mathbf{w}_{M, L}\right\|^{2}=1 .
\end{aligned}
$$

\subsection{Closed-form solution via convex optimization}

In order to obtain the optimal beamforming vectors $\mathbf{w}_{1,1}^{\star}, \ldots, \mathbf{w}_{M, L}^{\star}$, the Lagrange dual function could be constructed as

$$
\begin{aligned}
& L\left(\mathbf{w}_{1,1}, \cdots, \mathbf{w}_{M, L}, \lambda_{1,1}, \cdots, \lambda_{M, L}\right) \\
& =\sum_{m=1}^{L} \sum_{k=1}^{M} \lambda_{k, m}\left(\mathbf{w}_{k, m}^{*} \mathbf{w}_{k, m}-1\right)+\sum_{m=1}^{L} \sum_{k=1}^{M} \mathrm{MSE}_{k, m},
\end{aligned}
$$

where $\lambda_{1,1}, \cdots, \lambda_{M, L}$ are the Lagrange multipliers. Based on the Lagrange dual problem, Karush-Kuhn-Tucker (KKT) conditions are given as following Equations 13 and 14:

$$
\mathbf{w}_{k, m}^{\star *} \mathbf{w}_{k, m}^{\star}=1 \cdots \text { Primal constraint, }
$$

$$
\begin{aligned}
& \frac{\partial}{\partial \mathbf{w}_{k, m}^{\star}} L\left(\mathbf{w}_{1,1}^{\star}, \cdots, \mathbf{w}_{M, L}^{\star}, \lambda_{1,1}^{\star}, \cdots, \lambda_{M, L}^{\star}\right) \\
& \quad=0 \quad \cdots \text { Gradient of Lagrangian vanishes. }
\end{aligned}
$$

Since the problem is convex and Slater's condition is satisfied, the KKT conditions provide the optimal solution for (11). We take derivatives of Equation 12 with respect to $\mathbf{w}_{1,1}, \ldots, \mathbf{w}_{M, L}$, individually and equate them to zero as

$$
\begin{aligned}
& \frac{\partial}{\partial \mathbf{w}_{k, m}^{\star}} L\left(\mathbf{w}_{1,1}^{\star}, \cdots, \mathbf{w}_{M, L}^{\star}, \lambda_{1,1}^{\star}, \cdots, \lambda_{M, L}^{\star}\right) \\
& =\lambda_{k, m} \mathbf{w}_{k, m}^{*}+\mathbf{w}_{k, m}^{*} \sum_{i=1}^{M} \varepsilon_{i, m}^{2} \mathbf{h}_{i, m}^{\left(\tau_{i, m}\right)} \mathbf{h}_{k, m}^{\left(\tau_{k, m}\right) *} \\
& \quad+\mathbf{w}_{k, m}^{*} \sum_{i=1}^{M}\left(1-\varepsilon_{k, m}^{2}\right) \\
& \quad+\mathbf{w}_{k, m}^{*} \sum_{j=1, j \neq m}^{L} \sum_{i=1}^{M} \frac{\eta_{i, j, m}}{\rho_{k, m}} \varepsilon_{i, j, m}^{2} \mathbf{g}_{i, j, m}^{\left(\tau_{i, j}\right)} \mathbf{g}_{i, j, m}^{\left(\tau_{i, j, m}\right)} \\
& \quad+\mathbf{w}_{k, m}^{*} \sum_{j=1, j \neq m}^{L} \sum_{i=1}^{M} \frac{\eta_{i, j, m}}{\rho_{k, m}}\left(1-\varepsilon_{i, j, m}^{2}\right) \\
& \quad-\varepsilon_{k, m} \mathbf{h}_{k, m}^{\left(\tau_{k, m}\right) *}+\frac{\mathbf{w}_{k, m}^{*}}{\rho_{k, m}}=0, \quad \text { for all } k \text { and } m .
\end{aligned}
$$

Then, the optimal beamforming vectors, $\mathbf{w}_{1,1}^{\star}, \ldots, \mathbf{w}_{M, L}^{\star}$, could be solved as

$$
\begin{aligned}
\mathbf{w}_{k, m}^{\star}= & \varepsilon_{k, m}\left[\left(\lambda_{k, m}^{\star}+\frac{1}{\rho_{k, m}}+\sum_{i=1}^{M}\left(1-\varepsilon_{i, m}^{2}\right)\right.\right. \\
& \left.+\sum_{j=1, j \neq m}^{L} \sum_{i=1}^{M} \frac{\eta_{i, j, m}}{\rho_{k, m}}\left(1-\varepsilon_{i, j, m}^{2}\right)\right) \mathbf{I}_{M} \\
& +\sum_{i=1}^{M} \varepsilon_{i, m}^{2} \mathbf{h}_{i, m}^{\left(\tau_{i, m}\right)} \mathbf{h}_{i, m}^{\left(\tau_{i, m}\right) *} \\
& \left.+\sum_{j=1, j \neq m}^{L} \sum_{i=1}^{M} \frac{\eta_{i, j, m}}{\rho_{k, m}} \varepsilon_{i, j, m}^{2} \mathbf{g}_{i, j, m}^{\left(\tau_{i, j}\right)} \mathbf{g}_{i, j, m}^{\left(\tau_{i, j}\right) *}\right]^{-1} \mathbf{h}_{k, m}^{\left(\tau_{k, m}\right)},
\end{aligned}
$$

where $\mathbf{I}_{M}$ is the identity matrix of size $M$.

Based on the closed-form expression in (16), we can see how users' different SNRs, INRs, temporal correlations, and channel feedback latencies influence the choice of optimal beamforming vectors. In particular, the ratio of INR to SNR, $\frac{\eta_{i, j, m}}{\rho_{k, m}}$, is important factor to construct optimal 
beamforming vector. At the very-low INR regime $\left(\frac{\eta_{i, j, m}}{\rho_{k, m}} \rightarrow\right.$ $0)$, the optimal beamforming vector, $\mathbf{w}_{k, m}^{\star}$, converges to that of the conventional MMSE beamforming derived for single-cell multiuser MIMO systems as in [17]. This confirms that the conventional MMSE beamforming for single-cell MIMO systems is a special case of the proposed generalized MMSE beamforming for multicell MIMO systems when INR $\ll$ SNR. In addition, when all users' SNRs and temporal correlations are also assumed to be the same, the optimal MMSE beamforming vector in (16) is simplified to similar expression as derived in [14-16] as $\mathbf{w}_{k, m}^{\star}=\left[\left(M \bar{\sigma}_{e}+\frac{M}{\bar{\rho}}\right) \mathbf{I}_{M}+\sum_{i=1}^{M} \mathbf{h}_{i, m}^{\left(\tau_{i, m}\right)} \mathbf{h}_{i, m}^{\left(\tau_{i, m}\right) *}\right]^{-1} \mathbf{h}_{k, m}^{\left(\tau_{k, m}\right)}$, where $\bar{\rho}$ and $\bar{\sigma}_{e}$ are the same average SNR and channel error of users. At the very-high INR regime $\left(\frac{\eta_{i, j, m}}{\rho_{k, m}} \rightarrow \infty\right)$, the optimal beamforming vector is constructed largely dominated by the interference from neighboring BSs. In a nutshell, the generalized MMSE beamforming vectors are effectively determined and dynamically balanced considering the overall status of multicell MIMO systems.

Finally, to calculate the Lagrange multipliers, we define a Hermit matrix $\Phi$ from (15) as

$$
\begin{aligned}
\Phi= & \left(\sum_{i=1}^{M}\left(1-\varepsilon_{i, m}^{2}\right)+\sum_{j=1, j \neq m}^{L} \sum_{i=1}^{M} \frac{\eta_{i, j, m}}{\rho_{k, m}}\left(1-\varepsilon_{i, j, m}^{2}\right)\right) \cdot \mathbf{I}_{M} \\
& +\sum_{i=1}^{M} \varepsilon_{i, m}^{2} \mathbf{h}_{i, m, m}^{\left(\tau_{i, m}\right)} \mathbf{h}_{i, m, m}^{\left(\tau_{i, m}\right) *}+\sum_{j=1, j \neq m}^{L} \sum_{i=1}^{M} \frac{\eta_{i, j, m}}{\rho_{k, m}} \varepsilon_{i, j, m}^{2} \mathbf{g}_{i, j, m}^{\left(\tau_{i, j, m}\right)} \mathbf{g}_{i, j, m}^{\left(\tau_{i, j, m}\right) *} .
\end{aligned}
$$

Then, the eigen decomposition of the matrix $\Phi$ is $\Phi=$ $\mathbf{U} \Lambda \mathbf{U}^{*}$. Note that $\lambda_{k, m}^{\star}$ from the KKT conditions is the optimal value satisfying the power constraint. Putting (15) to the power constraint gives

$$
\begin{aligned}
\mathbf{w}_{k, m}^{\star *} \mathbf{w}_{k, m}^{\star} & \varepsilon_{k, m}^{2} \mathbf{h}_{k, m, m}^{\left(\tau_{k, m}\right) *}\left[\left(\lambda_{k, m}+\frac{1}{\rho_{k, m}}\right) \mathbf{I}_{M}+\Phi\right]^{-1} \\
& \times\left[\left(\lambda_{k, m}+\frac{1}{\rho_{k, m}}\right) \mathbf{I}_{M}+\Phi\right]^{-1} \mathbf{h}_{k, m, m}^{\left(\tau_{k, m}\right)} \\
= & \varepsilon_{k, m}^{2} \mathbf{h}_{k, m, m}^{\left(\tau_{k, m}\right) *} \mathbf{U}\left[\left(\lambda_{k, m}+\frac{1}{\rho_{k, m}}\right) \mathbf{I}_{M}+\Lambda\right]^{-1} \\
& \times\left[\left(\lambda_{k, m}+\frac{1}{\rho_{k, m}}\right) \mathbf{I}_{M}+\Lambda\right]^{-1} \mathbf{U}^{*} \mathbf{h}_{k, m, m}^{\left(\tau_{k, m}\right)} \\
= & \varepsilon_{k, m}^{2} \sum_{j=1}^{M} \frac{\left|\mathbf{h}_{k, m, m}^{\left(\tau_{k, m}\right) *} \mathbf{u}_{j}\right|^{2}}{\left(\lambda_{k, m}+\frac{1}{\rho_{k, m}}+\mu_{j}\right)^{2}},
\end{aligned}
$$

where $\mu_{j}$ is the $j$ th singular value of matrix $\Phi$ and $\mathbf{u}_{j}$ is the $j$ th column vector of matrix $\mathbf{U}$. The value of $\lambda_{k, m}^{\star}$ can be found solving (18). Now, all the beamforming vectors with Lagrange multipliers could be solved out.

\subsection{Impacts of multiple receive antennas}

Multiple receive antennas provide additional degree-offreedom for beamforming construction, which enables several variants of receiver beamforming. For simplicity, we try to limit our interests to the case of the receivers with maximal ratio combining, i.e., matched filters. The matched filter, $\mathbf{r}_{k, m}^{*}=\mathbf{w}_{k, m}^{*} \mathbf{h}_{k, m}$, combines received signals at multiple receive antennas, maximising received SNR. Since this SNR improvement is statistically independent with interference links, it reduces the contributions of the interference links in steering transmit beamforming vectors as seen from the analysis of the previous section. In the context of a multicell environment, increasing number of receive antennas can be effectively regarded as $\frac{\eta_{i, j, m}}{\rho_{k, m}} \rightarrow 0$, i.e., diminishing INR; the intercell interference becomes negligible in the limit of receive antenna number. In short, increasing number of receive antennas has an impact on the proposed MMSE beamforming in a way that beamforming vector constructions become gradually robust against interference links.

\section{Numerical results}

In this section, we present numerical results to evaluate the proposed generalized MMSE beamforming in terms of BER and average throughput. Simulation parameters are set based on commercialized LTE systems [10] and briefly listed in Table 1 . MSs are randomly dropped within cells. Simulation results are averaged over 1,000 independent channel realizations and user distributions. Different beamforming strategies for multicell MIMO systems such as MAX-SNR, MIN-GI, and MAX-SGINR are compared with the proposed generalized beamforming in terms of BER and average throughput performances.

Figure 2 shows how BER performance varies with BS transmit power. We assume a QPSK modulation and compute an average uncoded BER. The simulation result indicates that the generalized MMSE beamforming provides the lowest BER in all regions of BS transmit power. And, the performance gap increases with the transmit power

Table 1 Simulation parameters

\begin{tabular}{lc}
\hline & Value \\
\hline Carrier frequency, $f_{C}$ & $2 \mathrm{GHz}$ \\
$B S$ transmit power, $P$ & $10 \sim 40 \mathrm{dBm}$ \\
Number of BS antennas, $M$ & $2 \sim 4$ \\
Number of neighboring cells, $L$ & 3 \\
Cell radius & $1,000 \mathrm{~m}$ \\
Intracell feedback latency & $8 \mathrm{~ms}$ \\
Backhaul link delay & $20 \mathrm{~ms}$ \\
Pathloss exponent, $\alpha$ & 3.7 \\
Noise power, $N_{0}$ & $-100 \mathrm{dBm}$ \\
MS mobility, $V$ & $4 \mathrm{~km} / \mathrm{h}$ \\
\hline
\end{tabular}




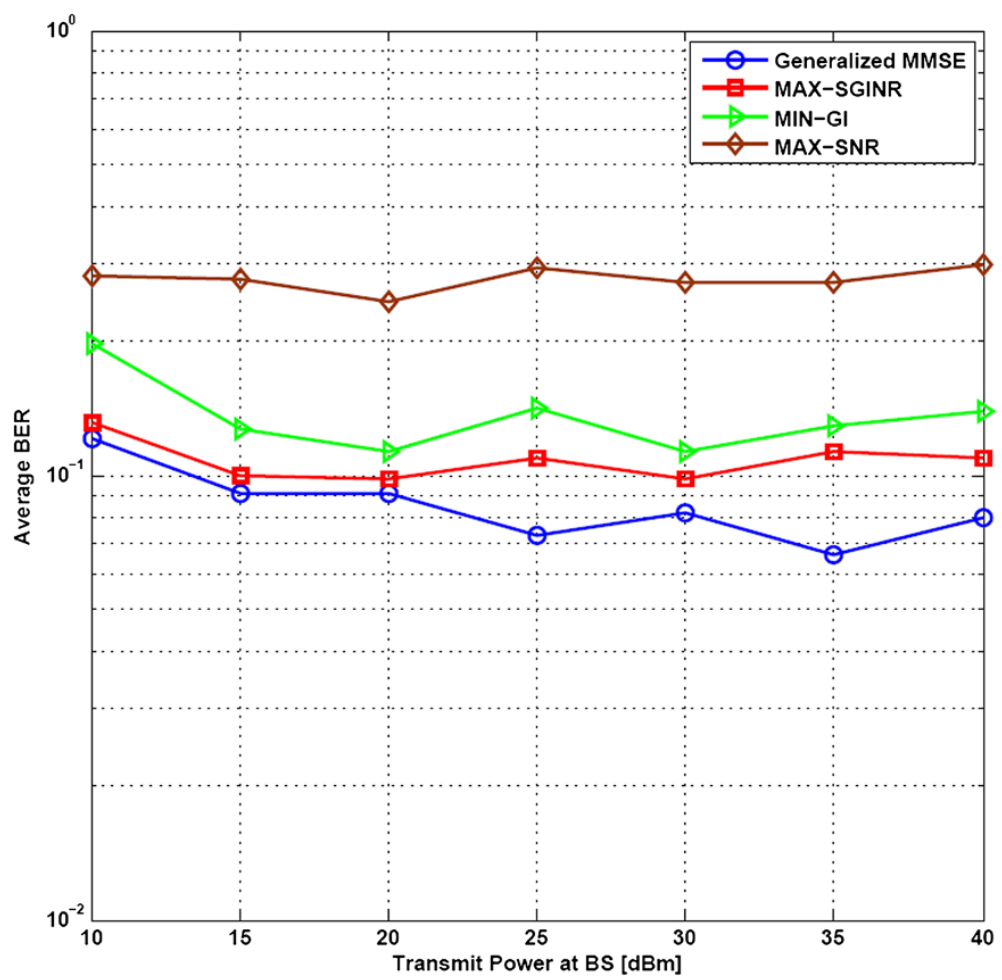

Figure 2 BER versus BS transmit power, where $L=3, M=4$, and $K=4$.

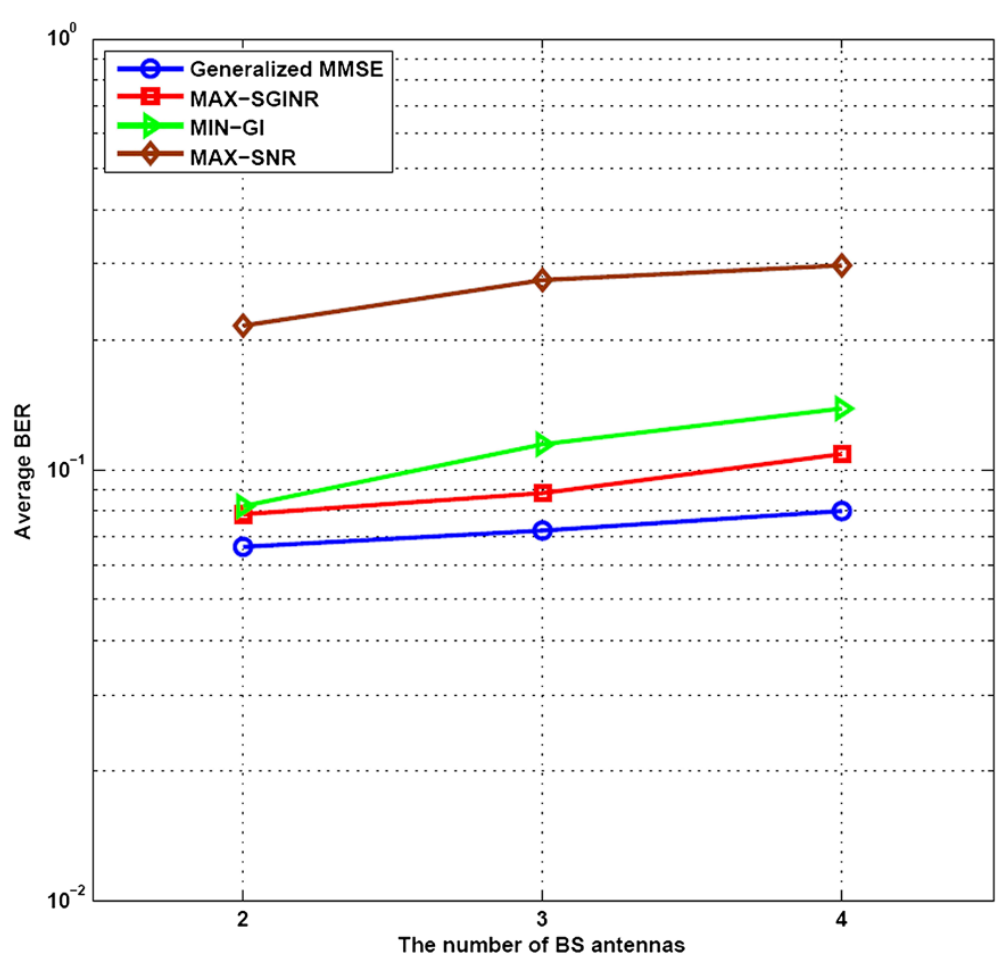

Figure 3 BER versus the number of $B S$ antennas, where $L=3$ and $P=40 \mathrm{dBm}$. 
since the generalized MMSE beamforming can effectively steer beamforming vectors considering both intracell and intercell interference in the interference-limited environments.

Figure 3 depicts how average BER varies with the number of BS antennas. As we can see from the figure, the generalized MMSE beamforming shows the lowest BER compared to MAX-SNR, MIN-GI, MAX-SGINR irrespective of the number of BS antennas. In particular, BER slightly increases with the number of BS antennas since the number of interfering signals also increases under the current setting that a user scheduler always selects maximum $M$ users at a time. The generalized MMSE beamforming keeps the degradation minimal by effectively adapting either types of interference, i.e., intracell and intercell interference.

Figure 4 compares average per-cell throughput performance of different beamforming strategies. As the transmit power at BS increases, the average per-cell throughput also increases. However, the average per-cell throughput eventually saturates in all beamforming strategies limited by interference. The generalized MMSE beamforming provides the considerable improvement regarding the saturation points. Specifically, the generalized MMSE beamforming provides $34 \%, 49 \%$, and $94 \%$ more throughput compared to MAX-SGINR, MIN-GI, and MAX-SNR beamforming, respectively.
On the other hand, Figure 5 focuses on average percell throughput in the $5 \%$ outage sense to show the performance of cell-edge users. As expected, the generalized MMSE beamforming achieves significantly higher throughput performance than MAX-SNR, MIN-GI, and MAX-SGINR beamforming in all regions of BS transmit power. In detail, the performance improvement of the generalized MMSE beamforming at the BS transmit power of $40 \mathrm{dBm}$ is $27 \%, 53 \%$, and $206 \%$ compared to MAX-SGINR, MIN-GI, and MAX-SNR beamforming, respectively. This improvement demonstrates that the generalized MMSE beamforming provides more benefits to users near the cell boundary since it can effectively mitigate intercell interference.

\section{Conclusions}

In this paper, we have proposed a generalized MMSE beamforming for downlink multicell MIMO systems with local CSI sharing and derived a closed-form solution. As the key novelty of this paper, we have considered a complicated but realistic multicell MIMO system model including the users' random geometries, different temporal correlations, and different channel feedback latencies. We have shown surprising improvements in terms of both BER and average throughput performances using the proposed generalized MMSE beamforming.

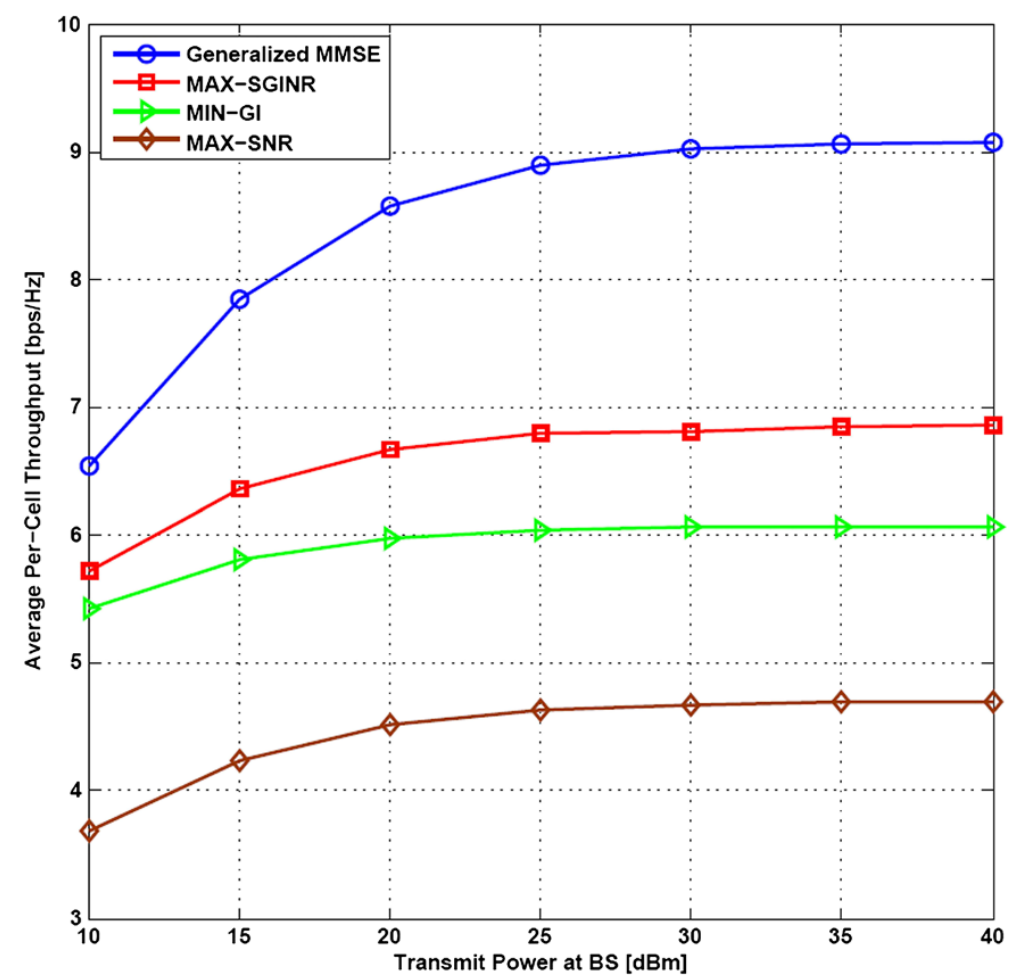

Figure 4 Average per-cell throughput versus BS transmit power, where $L=3, M=4$, and $K=4$. 


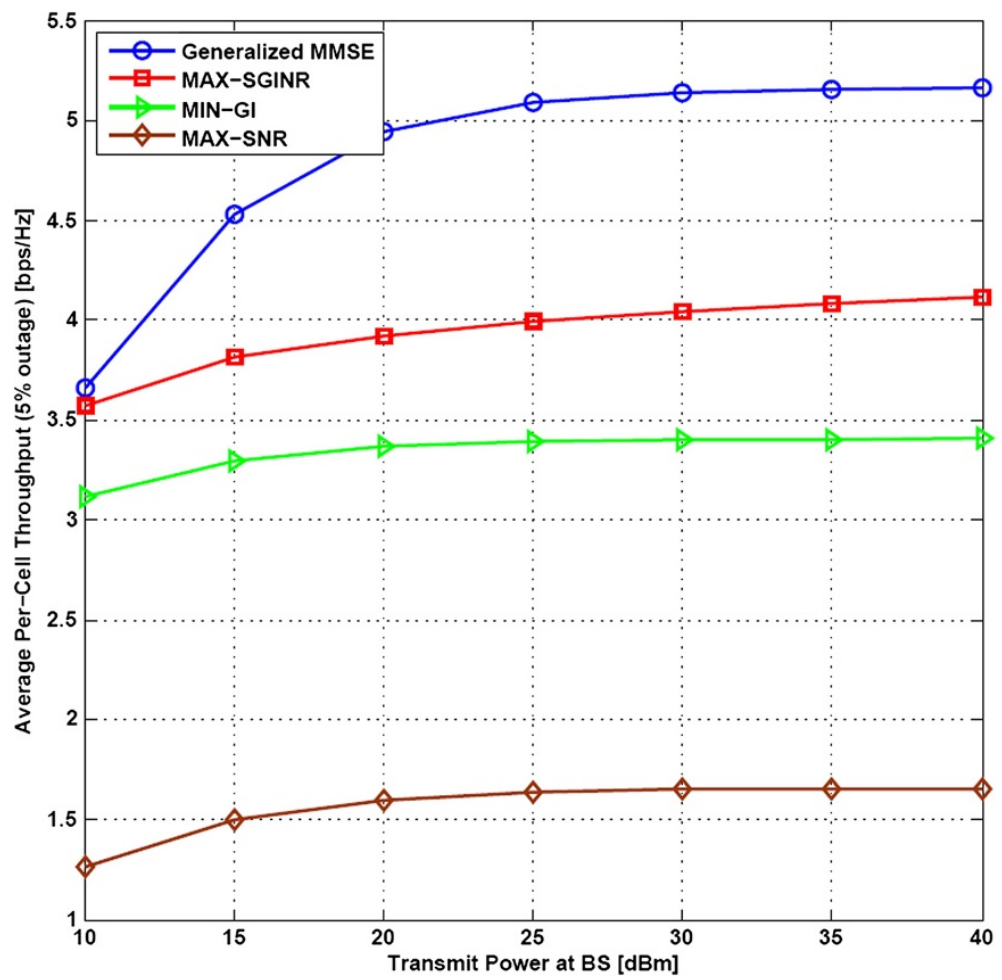

Figure 5 Average per-cell throughput in the $5 \%$ outage sense versus transmit power at $\mathrm{BS}$, where $L=3, M=4$, and $K=4$.

\section{Appendix}

\section{Proof of Theorem 1}

To derive (10), note that $x_{k, m}$ is i.i.d. circularly symmetric complex Gaussian random variables with zero mean and unit variance. Expectations are performed with respect to $x_{k, m}, \mathbf{h}_{w}, \mathbf{g}_{w}$, and are conditioned by $\varepsilon_{k, m}, \varepsilon_{k, m, j}, \rho_{k, m}, \eta_{k, m, j}, \mathbf{h}_{k, m}^{\left(\tau_{k, m}\right)}, \mathbf{g}_{k, m, j}^{\left(\tau_{k, m}\right)}$, which are omitted below for notational simplicity.

$$
\begin{aligned}
\mathbb{E} & {\left[\frac{1}{\rho_{k, m}}\left|y_{k, m}-\sqrt{\rho_{k, m}} \cdot x_{k, m}\right|^{2}\right] } \\
= & \mathbb{E}\left[\frac{1}{\rho_{k, m}}\left|y_{k, m}\right|^{2}\right]-\mathbb{E}\left[\frac{1}{\sqrt{\rho_{k, m}}} x_{k, m} y_{k, m}^{*}\right] \\
& -\mathbb{E}\left[\frac{1}{\sqrt{\rho_{k, m}}} x_{k, m}^{*} y_{k, m}\right]+\mathbb{E}\left[\left|x_{k, m}\right|^{2}\right] \\
\stackrel{(a)}{=} & \mathbb{E}\left[\left(\mathbf{w}_{k, m}^{*} \mathbf{h}_{k, m} \mathbf{h}_{k, m}^{*} \mathbf{w}_{k, m}\right)\right]+\mathbb{E}\left[\sum_{i=1, i \neq k}^{M}\left(\mathbf{w}_{i, m}^{*} \mathbf{h}_{k, m} \mathbf{h}_{k, m}^{*} \mathbf{w}_{i, m}\right)\right] \\
& +\mathbb{E}\left[\frac{1}{\rho_{k, m}} \sum_{j=1, j \neq m}^{L} \sum_{i=1}^{M} \eta_{k, m, j}\left(\mathbf{w}_{i, j}^{*} \mathbf{g}_{k, m, j} \mathbf{g}_{k, m, j}^{*} \mathbf{w}_{i, j}\right)\right] \\
& +\frac{\mathbf{w}_{k, m}^{*} \mathbf{w}_{k, m}}{\rho_{k, m}} \mathbb{E}\left[n_{k, m}^{*} n_{k, m}\right]-\mathbb{E}\left[\left(\mathbf{w}_{k, m}^{*} \mathbf{h}_{k, m}\right)\right] \\
& -\mathbb{E}\left[\left(\mathbf{h}_{k, m}^{*} \mathbf{w}_{k, m}\right)\right]+1,
\end{aligned}
$$

$$
\begin{aligned}
\stackrel{(b)}{=} & \varepsilon_{k, m}^{2} \cdot \mathbf{w}_{k, m}^{*} \mathbf{h}_{k, m}^{\left(\tau_{k, m}\right)} \mathbf{h}_{k, m}^{\left(\tau_{k, m}\right) *} \mathbf{w}_{k, m}+\left(1-\varepsilon_{k, m}^{2}\right) \cdot \mathbf{w}_{k, m}^{*} \mathbf{w}_{k, m} \\
& +\varepsilon_{k, m}^{2} \cdot \sum_{j=1, j \neq k}^{M} \mathbf{w}_{j, m}^{*} \mathbf{h}_{j, m}^{\left(\tau_{j, m}\right)} \mathbf{h}_{j, m}^{\left(\tau_{j, m}\right) *} \mathbf{w}_{j, m}+\left(1-\varepsilon_{k, m}^{2}\right) \\
& \times \sum_{j=1, j \neq k}^{M} \mathbf{w}_{j, m}^{*} \mathbf{w}_{j, m}+\frac{1}{\rho_{k, m}} \\
& \times \sum_{j=1, j \neq m}^{L} \sum_{i=1}^{M} \varepsilon_{k, m, j}^{2} \cdot \eta_{k, m, j} \cdot \mathbf{w}_{i, j}^{*} \mathbf{g}_{i, j, m}^{\left(\tau_{i, j}\right)} \mathbf{g}_{i, j, m}^{\left(\tau_{i, j}\right) *} \mathbf{w}_{i, j} \\
& +\frac{1}{\rho_{k, m}} \cdot \sum_{j=1, j \neq m}^{L} \sum_{i=1}^{M}\left(1-\varepsilon_{k, m, j}^{2}\right) \cdot \eta_{k, m, j} \cdot \mathbf{w}_{i, j}^{*} \mathbf{w}_{i, j} \\
& +\frac{\mathbf{w}_{k, m}^{*} \mathbf{w}_{k, m}}{\rho_{k, m}}-\varepsilon_{k, m} \cdot \mathbf{w}_{k, m}^{*} \mathbf{h}_{k, m}^{\left(\tau_{k, m}\right)}-\varepsilon_{k, m} \cdot \mathbf{h}_{k, m}^{\left(\tau_{k, m}\right) *} \mathbf{w}_{k, m}+1,
\end{aligned}
$$

where $(a)$ uses the fact that $\left\|\mathbf{w}_{k, m}^{*}\right\|^{2}=1, \mathrm{E}\left[\left|x_{k, m}^{*} x_{i, j}\right|^{2}\right]=$ 1 only when $k=i$ and $m=j, \mathrm{E}\left[\left|x_{k, m}{ }^{*} x_{i, j}\right|^{2}\right]=0$ otherwise, and $(b)$ computes the conditional expectation with respect to $\mathbf{h}_{\mathrm{w}}$ and $\mathbf{g}_{\mathrm{w}}$ after replacing $\mathbf{h}_{k, m}$ and $\mathbf{g}_{k, m, j}$ with (2) and (3), respectively. In this computation, we also use the assumption that the elements of $\mathbf{h}_{\mathrm{w}}$ and $\mathbf{g}_{\mathrm{w}}$ are i.i.d. circularly symmetric complex Gaussian random variables 
with zero mean and unit variance, i.e., $\mathbb{E}_{\mathbf{h}_{\mathrm{w}}}\left[\mathbf{h}_{\mathrm{w}} \mathbf{h}_{\mathrm{w}}{ }^{*}\right]=\mathrm{I}_{M}$ and $\mathbb{E}_{\mathbf{g}_{\mathrm{w}}}\left[\mathbf{g}_{\mathrm{w}} \mathbf{g}_{\mathrm{w}}{ }^{*}\right]=\mathrm{I}_{M}$. This finally gives (10).

\section{Competing interests}

The authors declare that they have no competing interests.

\section{Acknowledgements}

This paper was supported by Samsung Electronics Co., Ltd. and the Brain Korea 21 Plus Project in 2014

\section{Author details}

${ }^{1}$ Department of Electronics Engineering, Gachon University, 1342 Seonnamdaero, Sugeong-gu, Seongnam-si, Gyeonggi-do 461-701, Korea. ${ }^{2}$ Samsung Electronics Co., Maetan 3-dong, Yeongtong-gu, Suwon-si, Gyeonggi-do, Korea. ${ }^{3}$ Department of Electrical and Computer Engineering and INMC, Seoul National University, 599 Gwanak-ro, Gwanak-Gu, Seoul 110-799, Korea.

Received: 10 October 2013 Accepted: 14 May 2014

Published: 24 May 2014

\section{References}

1. RS Blum, MIMO capacity with interference. IEEE J. Sel. Areas Commun. 21(5), 793-801 (2003)

2. JG Andrews, W Choi, RW Heath, Overcoming interference in spartial multiplexing MIMO wireless networks. IEEE Wireless Commun. Mag. 14(6), 95-104 (2007)

3. D Gesbert, S Hanly, H Huang, S Shamai, O Simenone, W Yu, Multi-cell MIMO cooperative networks: a new look at interference. IEEE J. Sel. Areas Commun. 28(9), 1380-1408 (2010)

4. S Shamai, AD Wyner, Information-theoretic considerations for symmetric, cellular, multiple-access fading channels. II. IEEE Trans. Inf. Theory. 43(6), 1895-1911 (1997)

5. MK Karakayali, GJ Foschini, RA Valenzuela, Network coordination for spectrally efficient communications in cellular systems. IEEE Wireless Commun. Mag. 13(4), 56-61 (2006)

6. I Sohn, SH Lee, JG Andrews, Belief propagation for distributed downlink beamforming in cooperative MIMO cellular networks. IEEE Trans. Wireless Commun. 10(12), 4140-4149 (2011)

7. E Visotsky, U Madhow, Optimum beamforming using transmit antenna arrays, in Proceedings of the IEEE Vehicular Technology Conference (Houston, July 1999)

8. KMH Zuleita, D Gesbert, Balancing egoism and altruism on interference channel: the MIMO case, in Proceedings of the IEEE International Conference on Communications (Cape Town, 23-27 May 2010)

9. BO Lee, HW Je, OS Shin, KB Lee, A novel uplink MIMO transmission scheme in a multicell environment. IEEE Trans. Wireless Commun. 8(10), 4981-4987 (2009)

10. 3rd Generation Partnership Project, LTE; Evolved Universal Terrestrial Radio Access (E-UTRA); Physical Channels and Modulation (3GPP TS 36.211 Std. version 11.1.0, Release 11), (ETSI, Sophia-Antipolis Cedex, 2013)

11. I Sohn, JG Andrew, KB Lee, MIMO broadcast channels with spatial heterogeneity. IEEE Trans. Wireless Commun. 9(8), 2449-2454 (2010)

12. D Gesbert, M Kountouris, Joint power control and user scheduling in multicell wireless networks: capacity scaling laws. IEEE Trans. Inf. Theory. 8(10), 4981-4987 (2011)

13. 3rd Generation Partnership Project; R1-071804: Reply LS to R3-070527/R1-071242 on Backhaul (X2 interface) Delay, Malta, 26-30 Mar 2007. http://www.3gpp.org/

14. AD Dabbagh, DJ Love, Multiple antenna MMSE based downlink precoding with quantized feedback or channel mismatch. IEEE Trans. Commun. 56(11), 1859-1868 (2008)

15. S Serbetli, A Yener, Tranceiver optimization for multiuser MIMO systems. IEEE Trans. Signal Process. 52(1), 214-226 (2004)

16. J Zhang, Y Wu, S Zhou, J Wang, Joint linear transmitter and receiver design for the downlink of multiuser MIMO systems. IEEE Commun. Lett. 9(11), 991-993 (2005)

17. HJ Lee, I Sohn, DH Kim, KB Lee, Generalized MMSE beamforming for downlink MIMO systems, in Proceedings of IEEE International Conference on Communications (ICC 2011) (Kyoto, September 5)

18. CC Tan, NC Beaulieu, On first-order Markov modeling for the Rayleigh fading channel. IEEE Trans. Commun. 48(12), 2032-2040 (2000)
19. EN Onggosanusi, A Gatherer, AG Dabak, S Hosur, Performance analysis of close-loop transmit diversity in the presence of feedback delay. IEEE Trans. Commun. 49(9), 1618-1630 (2001)

20. N Jindal, MIMO broadcast channels with finite rate feedback. IEEE Trans. Inf. Theory. 52(7), 2080-2089 (2009)

21. T Yoo, N Jindal, A Goldsmith, Multi-antenna downlink channels with limited feedback and user selection. IEEE J. on Sel. Areas Commun. 25(7), 1478-1491 (2007)

doi:10.1186/1687-1499-2014-86

Cite this article as: Sohn et al:: Generalized MMSE beamforming for multicell MIMO systems with random user geometry and channel feedback latency. EURASIP Journal on Wireless Communications and Networking 2014 2014:86.

\section{Submit your manuscript to a SpringerOpen ${ }^{\mathcal{O}}$ journal and benefit from:}

- Convenient online submission

- Rigorous peer review

- Immediate publication on acceptance

- Open access: articles freely available online

- High visibility within the field

- Retaining the copyright to your article

Submit your next manuscript at $\boldsymbol{~ s p r i n g e r o p e n . c o m ~}$ 